\title{
THE EXISTENCE OF $Q$-SETS IS EQUIVALENT TO THE EXISTENCE OF STRONG $Q$-SETS
}

\author{
TEODOR C. PRZYMUSIŃSKI
}

\begin{abstract}
In this note we prove that the existence of an uncountable $Q$-set is equivalent to the existence of an uncountable strong $Q$-set, i.e. a $Q$-set all finite powers of which are $Q$-sets.
\end{abstract}

A $Q$-set is a separable metric space all subsets of which are $F_{\sigma}$ sets. It is well known that the existence of an uncountable $Q$-set is equivalent to the existence of a normal separable nonmetrizable Moore space and is undecidable in ZFC (for more information on $Q$-sets see the survey paper [F1]).

A strong $Q$-set is a $Q$-set all finite powers of which are $Q$-sets. It is known that the Pixley-Roy hyperspace of a metric separable space $M$ is a normal nonmetrizable Moore space if and only if $M$ is an uncountable strong $Q$-set [PT], [R].

W. G. Fleissner proved that the square of a $Q$-set in general does not have to be a $Q$-set [F2]. We prove that the existence of an uncountable $Q$-set is equivalent to the existence of an uncountable strong $Q$-set. ${ }^{1}$ We also formulate some other statements equivalent to the existence of uncountable $Q$-sets.

LEMMA 1. If $\left\{\sigma_{n}\right\}_{n=1}^{\infty}$ is a sequence of separable metrics $\sigma_{n}$ on $X$ then there exists a separable metric $\sigma$ on $X$ which is stronger than any of the metrics $\sigma_{n}$.

Proof. Consider the diagonal of $\prod_{n=1}^{\infty}\left(X, \sigma_{n}\right)$.

Lemma 2 (cf. [BBM, Theorem 3]). Let $A$ be a subset of $X \times Y$ and $\rho$ a separable metric on $X$. All horizontal sections of $A$ (i.e. sets $A_{y}=\{x \in X:(x, y) \in A\}$ for $y \in Y)$ are $F_{\sigma}$ subsets of $(X, \rho)$ if and only if there exists a separable metric $\sigma$ on $Y$ such that $A$ is an $F_{\sigma}$ subset of $(X, \rho) \times(Y, \sigma)$.

Proof. The "if" implication is obvious. Suppose that all horizontal sections $A_{y}$ of $A$ are $F_{\sigma}$ subsets of $(X, \rho)$. For every $y \in Y$ let $X \backslash A_{y}=\cap_{n=1}^{\infty} G_{y, n}$, where sets $G_{y, n}$ are open in $(X, \rho)$ and let $\left\{B_{m}\right\}_{m=1}^{\infty}$ be a base for $(X, \rho)$. Put $C_{n, m}=\{y \in Y$ : $\left.B_{m} \subset G_{y, n}\right\}$.

One easily verifies that

$$
(X \times Y) \backslash A=\bigcap_{n=1}^{\infty} \bigcup_{m=1}^{\infty}\left(B_{m} \times C_{n, m}\right) .
$$

It suffices to find a separable metric $\sigma$ on $Y$ in which all sets $C_{n, m}$ are open (cf. Lemma 1).

Presented to the Society, August 7, 1978; received by the editors March 26, 1979. AMS (MOS) subject classifications (1970). Primary 02K05, 54E30; Secondary 02K30, 54E35.

${ }^{1}$ This answers a question brought to my attention by F. D. Tall. 
Let us denote by $R$ the real line or any other set of cardinality continuum. As usual $\omega_{1}=\left\{\alpha: \alpha<\omega_{1}\right\}$.

THEOREM. The following conditions are equivalent:

(i) there exists an uncountable $Q$-set;

(ii) there exists an uncountable strong $Q$-set;

(iii) $2^{\omega_{1}}=2^{\omega}$ and for every subset $A$ of $R \times \omega_{1}$ there exist separable metrics $\rho$ on $R$ and $\sigma$ on $\omega_{1}$ such that $A$ is an $F_{\sigma}$ subset of $(R, \rho) \times\left(\omega_{1}, \sigma\right)$;

(iv) $2^{\omega_{1}}=2^{\omega}$ and for every family $Q$ of $\omega_{1}$ subsets of $R$ there exists a separable metric $\rho$ on $R$ such that all members of $\mathbb{Q}$ are $F_{\sigma}$ subsets of $(R, \rho)$;

(v) $2^{\omega_{1}}=2^{\omega}$ and for every $n<\omega$ and every subset $A$ of $R \times \omega_{1}^{n}$ there exist separable metrics $\rho$ on $R$ and $\sigma$ on $\omega_{1}$ such that $A$ is an $F_{\sigma}$ subset of $(R, \rho) \times\left(\omega_{1}, \sigma\right)^{n}$;

(vi) $2^{\omega_{1}}=2^{\omega}$ and for every $n<\omega$ and every family $\mathcal{Q}$ of $\omega_{1}$ subsets of $R \times \omega_{1}^{n}$ there exist separable metrics $\rho$ on $R$ and $\sigma$ on $\omega_{1}$ such that all members of $\mathcal{Q}$ are $F_{\sigma}$ subsets of $(R, \rho) \times\left(\omega_{1}, \sigma\right)^{n}$.

REMARK 1. W. G. Fleissner proved that there exist models of set theory in which there exist $Q$-sets of cardinality $\omega_{2}$, but in which there are no strong $Q$-sets of cardinality $\omega_{2}$ [F2]. This implies that conditions (i) and (ii) in the above theorem are no longer equivalent if one assumes that the considered $Q$-sets are of cardinality $\omega_{2}$. Similarly, conditions (iii) and (v) and conditions (iv) and (vi) are not equivalent if one replaces $\omega_{1}$ by $\omega_{2}$.

REMARK 2. Conditions (i)-(vi) above are also equivalent to the following propositions (for more information, see [P]):

(vii) $R^{\omega_{1}}$ is a continuous image of a separable first countable space (here $R$ carries its usual topology);

(viii) every space of cardinality (or weight) $\omega_{1}$ can be embedded into a sequentially separable space.

Proof of THE TheOREM. (i) $\rightarrow$ (iii). Let $\sigma$ be a separable metric on $\omega_{1}$ such that $\left(\omega_{1}, \sigma\right)$ is a $Q$-set. From Lemma 2 it follows that there exists a separable metric $\rho$ on $R$ such that $A$ is an $F_{\sigma}$ subset of $(R, \rho) \times\left(\omega_{1}, \sigma\right)$.

(iii) $\rightarrow$ (iv). Let $\mathbb{Q}=\left\{A_{\alpha}: \alpha<\omega_{1}\right\}$. It suffices to put $A=\cup\left\{A_{\alpha} \times\{\alpha\}: \alpha<\right.$ $\left.\omega_{1}\right\} \subset R \times \omega_{1}$ and apply (iii).

(iv) $\rightarrow$ (v). For the sake of simplicity, we shall prove (v) only in the case of $n=2$. The general case can be similarly proved by induction.

Let $A$ be a subset of $R \times \omega_{1} \times \omega_{1}$ and put $B=\{(r, \alpha, \beta) \in A: \alpha<\beta\}$ and $C=\{(r, \alpha, \beta) \in A: \alpha \geqslant \beta\}$. From the symmetry of assumptions and the equality $A=B \cup C$ we infer that without loss of generality we can assume that $A=B$. For every $\beta \in \omega_{1}$ put $A_{\beta}=\left\{(r, \alpha) \in R \times \omega_{1}:(r, \alpha, \beta) \in A\right\}$. From Lemma 2 it follows that it suffices to show that there exist separable metrics $\rho$ on $R$ and $\sigma$ on $\omega_{1}$ such that all sets $A_{\beta}$ are $F_{\sigma}$ subsets of $(R, \rho) \times\left(\omega_{1}, \sigma\right)$ for $\beta \in \omega_{1}$. Since $A_{\beta} \subset R \times$ $(\beta+1)$, for every $\beta<\omega_{1}$, it suffices to show that there exists a separable metric $\rho$ on $R$ such that all sets $A_{\beta \alpha}=\left\{r \in R:(r, \alpha) \in A_{\beta}\right\}$ are $F_{\sigma}$ subsets of $(R, \rho)$ for $\beta<\omega_{1}$ and $\alpha \leqslant \beta$, but this is a consequence of (iv).

(v) $\rightarrow$ (vi). Let $\mathbb{Q}=\left\{A_{\alpha}: \alpha<\omega_{1}\right\}$. It suffices to put $A=\cup\left\{A_{\alpha} \times\{\alpha\}: \alpha<\omega_{1}\right\}$ $\subset R \times \omega_{1}^{n+1}$ and apply (v). 
(vi) $\rightarrow$ (ii). It is enough to prove that for every $n<\omega$ there exists a separable metric $\sigma_{n}$ on $\omega_{1}$ such that $\left(\omega_{1}, \sigma_{n}\right)^{n}$ is a $Q$-set, because then, by Lemma 1 , there would exist a $\sigma$ which is stronger than any of the metrics $\sigma_{n}$ and clearly $\left(\omega_{1}, \sigma\right)$ is a strong $Q$-set.

Let $n<\omega$ and let $\left\{A_{r}: r \in R\right\}$ be the family of all subsets of $\omega_{1}^{n}$. Put $A=$ $\cup\left\{\{r\} \times A_{r}: r \in R\right\} \subset R \times \omega_{1}^{n}$. By (vi) there exist separable metrics $\rho$ on $R$ and $\sigma$ on $\omega_{1}$ such that $A$ is an $F_{\sigma}$ subset of $(R, \rho) \times\left(\omega_{1}, \sigma\right)^{n}$. Clearly, $\left(\omega_{1}, \sigma\right)^{n}$ is a $Q$-set.

Remark 3. R. Pol suggested a different proof of the equivalence of (i) and (ii) based on the fact that $\omega_{1}^{2}$ is a union of countably many graphs and inverse graphs.

\section{REFERENCES}

[BBM] R. H. Bing, W. W. Bledsoe and R. D. Mauldin, Sets generated by rectangles, Pacific J. Math. 51 (1974), 27-36.

[F1] W. G. Fleissner, Current research on Q-sets, Proc. Bolyai Janos Colloq. on Topology (Budapest, 1978) (to appear).

[F2] , Squares of $Q$-sets (to appear).

[P] T. C. Przymusinski, On the equivalence of certain set theoretic and topological conditions, Proc. Bolyai Janos Colloq. on Topology (Budapest, 1978) (to appear).

[PT] T. C. Przymusinski and F. D. Tall, The undecidability of the existence of a nonseparable normal Moore space satisfying the countable chain condition, Fund. Math. 85 (1974), 291-297.

[R] M. E. Rudin, Pixley-Roy and the Souslin line, Proc. Amer. Math. Soc. 79 (1979), 128-134.

Instytut Matematyczny, PAN, Warsaw 00-950, Śniadeckich 8, Poland 